\title{
Electron transport in single wall carbon nanotube weak links in the Fabry-Perot regime
}

\author{
H. I. Jørgensen $\dagger, 1$, 冈 K. Grove-Rasmussen $\dagger^{1}{ }^{1}$ T. Novotný, ${ }^{1,2}$ K. Flensberg, ${ }^{1}$ and P. E. Lindelof ${ }^{1}$ \\ ${ }^{1}$ Nano-Science Center, Niels Bohr Institute, University of Copenhagen, \\ Universitetsparken 5, DK-2100 Copenhagen Ø, Denmark \\ ${ }^{2}$ Department of Electronic Structures, Faculty of Mathematics and Physics, \\ Charles University, Ke Karlovu 5, 12116 Prague, Czech Republic \\ $\dagger$ These authors contributed equally to this work
}

(Dated: November 5, 2018)

\begin{abstract}
We fabricated reproducible high transparency superconducting contacts consisting of superconducting $\mathrm{Ti} / \mathrm{Al} / \mathrm{Ti}$ trilayers to gated single-walled carbon nanotubes (SWCNTs). The reported semiconducting SWCNT have normal state differential conductance up to $3 e^{2} / h$ and exhibit clear FabryPerot interference patterns in the bias spectroscopy plot. We observed subharmonic gap structure in the differential conductance and a distinct peak in the conductance at zero bias which is interpreted as a manifestation of a supercurrent. The gate dependence of this supercurrent as well as the excess current are examined and compared to a coherent theory of superconducting point contacts with good agreement.

PACS numbers: 74.45.+c, 73.23.Ad, 73.63.Fg, 74.50.+r
\end{abstract}

Electron transport through a SWCNT bridging two metal electrodes has been studied intensively over the last years. At low temperatures different transport regimes depending on the transparency of the metal-SWCNT interfaces have been identified. With low transparency contacts a quantum dot (QD) will be defined in the SWCNT [1, 2, 3, 4], and with intermediate transparency contacts Kondo resonances around zero bias are observed [5, [6]. High transparency contacts are in recent years also reported, where the SWCNT constitutes an electron waveguide with Fabry-Perot (FP) interferences [7, 8].

Changing the metal electrodes to superconductors (SC) dramatically changes the transport characteristics. In such junctions the carbon nanotube (CNT) forms a weak link between the two superconductors and several interesting effects can be observed. At zero bias a supercurrent can flow through the weak link 9, 10, 11] due to the Josephson effect [12], while at low biases current will be carried by Multiple Andreev reflections (MAR) at the two CNT-S interfaces [13, 14, 15]. For large bias, these effects will give rise to an excess current.

In fact, only very recently have these effects been seen in SWCNT devices 9] similar to the one presented here. In this Letter, we present transport measurements on a gated S-SWCNT-S Josephson junction at low temperatures, with high transparency contacts. We focus on the gate dependence of the excess current and zero-bias conductance peak in the FP regime.

The SWCNTs are grown by chemical vapor deposition (CVD) from catalyst islands made by electron beam lithography and positioned relative to predefined alignment marks. The details of the CVD-growth procedure are described elsewhere [16, 17]. After growth, source and drain electrodes consisting of superconducting trilayers are positioned next to the catalyst islands to con- tact the SWCNT. The gap between the source and drain trilayer films is approximately $500 \mathrm{~nm}$. The superconducting trilayers consist of $5 \mathrm{~nm}$ titanium to make good contact to the SWCNT, then of $40 \mathrm{~nm}$ aluminum to raise the transition temperature, and finally $5 \mathrm{~nm}$ titanium to stop oxidation of the aluminum.

Our devices are made on a highly doped silicon wafer with a $0.5 \mu \mathrm{m}$ thermally oxidized $\mathrm{SiO}_{2}$ layer on top. We use the silicon substrate as a back gate. To be able to measure the transition temperature $T_{C}$ and the critical field $B_{C}$ of the trilayer films at low temperatures we furthermore define a four probe device of the superconducting trilayer. For the device in this Letter we find $T_{C}=750 \mathrm{mK}, B_{C}=75 \mathrm{mT}$ and from BCS theory we calculate a superconducting energy gap of $2 \Delta=3.5 k_{B} T_{C}=230 \mu \mathrm{eV}$. However, the actual effective value of $\Delta$ for the CNT weak link might differ from this measured value due to interface effects and, indeed, we found from the fit of the excess current measurements (see below) that the effective gap is reduced by about a factor of $\sim 0.7$. All measurements are preformed at $300 \mathrm{mK}$ in a sorption pumped ${ }^{3} \mathrm{He}$ cryostat (Oxford Instruments Heliox). The measurements are made with standard DAQ cards, lock-in amplifiers (excitation $5 \mu \mathrm{V}$ ), and opto-couplers to reduce noise.

Fig. 11 shows a gatesweep from $-10 \mathrm{~V}$ to $0 \mathrm{~V}$ with $V_{s d}=1 \mathrm{mV}(>2 \Delta / e)$. It displays strong gate dependence: High conductance at high negative gate voltages and low conductance at small gate voltages, which indicates that the SWCNT is semiconducting. Addition of the first hole and first electron cannot be identified, probably due to substrate perturbations. The SWCNT defines a QD with gate depended Schottky barriers at each interface. The gate thus tunes both the energy levels of the QD and the strength of the Schottky barriers. 


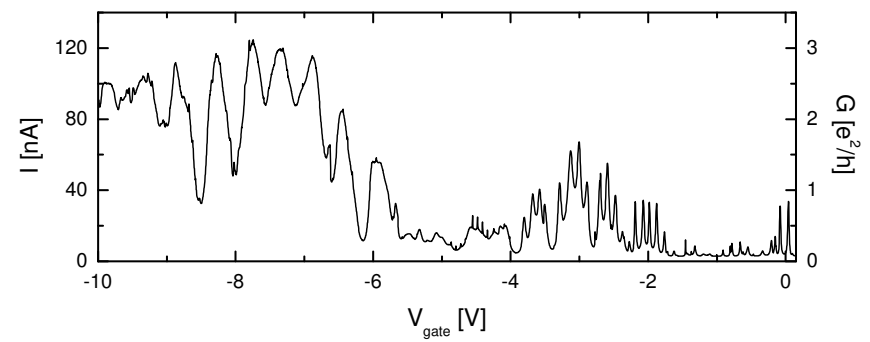

FIG. 1: Current and conductance for the S-SWCNT-S Josephson junction as a function of voltage applied to the back gate with $1 \mathrm{mV}$ source drain voltage. The high conductance regime with Fabry-Perot oscillations is reached at large negative gate voltages $(<-6 \mathrm{~V})$. Coulomb blockade peaks are seen at lower gate voltages.

From $V_{\text {gate }} \sim-4 \mathrm{~V}$ to $V_{\text {gate }} \sim-2 \mathrm{~V}$ the Schottky barriers are large and the SWCNT constitutes a closed QD, i.e., the charging energy $U_{C}$ is larger than the broadening of the energy levels $\Gamma$. Transport is here dominated by charging effects and Coulomb blockade peaks are clearly visible. Some of the peaks are spaced into periods of four due the four-fold degeneracy (spin and orbital) of each energy level, also confirmed by bias spectroscopy plots (not shown). Such characteristic is sign of a high quality SWCNT. As the gate voltage is decreased to more negative values the Schottky barriers are decreased, leading to an increase of $\Gamma$. Below $V_{\text {gate }} \sim-5 \mathrm{~V}$ the dot opens, $\Gamma$ becomes larger than $U_{C}$, and charging effects of the QD are no longer dominant. Instead FP interference of the electron waves being reflected at the SWCNT-electrode interfaces dominates transport.

Fig. 2(a) shows a bias spectroscopy plot in this gate region with a small magnetic field applied $(B=100 \mathrm{mT})$ to suppress the superconducting state of the electrodes. The average differential conductance in this gate region is around $\sim 2.5 e^{2} / h$ with maximums of about $\sim 3 e^{2} / h$, approaching the theoretical limit of $4 e^{2} / h=(6.5 \mathrm{k} \Omega)^{-1}$. The maximum value of $\sim 3 e^{2} / h$ of the conductance implies a rather large asymmetry $\Gamma_{L} / \Gamma_{R} \approx 5$ of the CNT couplings to the contacts (found from a resonant level model representing the system well around a conductance peak), in contrast to fairly symmetric couplings reported elsewhere [9, 14]. As $V_{\text {gate }}$ and $V_{\text {sd }}$ are changed the dips in conductance evolve into straight lines, forming a mesh of crossing dark lines. These pronounced oscillations in differential conductance versus $V_{\text {gate }}$ and $V_{\text {sd }}$ are clear signs of the FP interferences [7].

As we turn off the magnetic field, i.e., turn on the superconducting state of the electrodes, an overall increase in differential conductance between $V_{\text {sd }} \sim \pm 2 \Delta / e$ is observed (Fig. 2(b)). A more detailed bias spectroscopy plot of this overall increase through two successive resonances is shown in Fig. 2(c). Detailed measurements

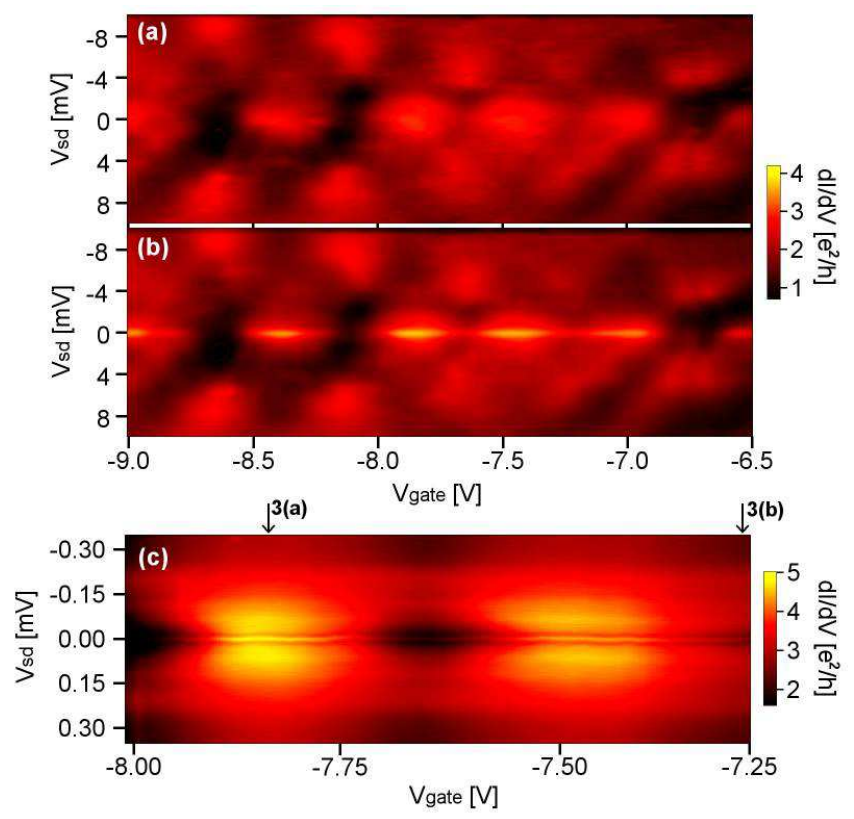

FIG. 2: (Color online) (a) Bias spectroscopy plot in the high transparency gate region, with a small magnetic field $(B=100 \mathrm{mT})$ to suppress the superconducting state of the electrodes. (b) Analogous to (a) but without magnetic field, i.e., with superconducting electrodes. (c) Close-up on two resonances from which the excess current and supercurrent of Fig. 4 are extracted. Arrows are pointing to the gate voltages where the graphs in Figs. [3] (a-b) are measured.

with lock-in amplifier of differential conductance versus $V_{\text {sd }}$ at gate voltages indicated in Fig. 2(c) are shown in Figs. 3(a-b), where (a) is a cut through a resonance and (b) is a cut through an antiresonance. A characteristic conductance variation between $V_{\text {sd }} \sim \pm 2 \Delta / e$ is seen for all gate voltages. Close to $\left|V_{\mathrm{sd}}\right| \sim 2 \Delta / e$ the conductance starts to increase while at smaller source drain voltages a dip centered around zero bias also develops. In Fig. 3(b) this dip can be seen between $V_{\text {sd }} \sim \pm 80 \mu \mathrm{V}$ and less strongly in Fig. [3(a). The change in conductance between $V_{\text {sd }} \sim \pm 2 \Delta / e$ to typically higher, but sometimes also lower values, than the normal state differential conductance $G_{N}$ is because superconductivity induced transport mechanisms occur.

Between $V_{\text {sd }} \sim \pm 2 \Delta / e$ transport is governed by Andreev reflections (ARs) [18 and normal reflections. An electron with energy $|\epsilon|<\Delta$ relative to the Fermi energy in the normal region has (depending on the barrier strength) a probability for being AR on the superconductor as a hole effectively transferring two electrons (one Cooper pair) through the NS interface 19, 20]. For $|\epsilon|>\Delta$ ARs are still possible but fall off rapidly. Multiple Andreev reflections between the two superconducting leads at finite bias give rise to a sub gap structure (SGS) 21, 22, 23, 24], while at zero bias a dissipationless super- 

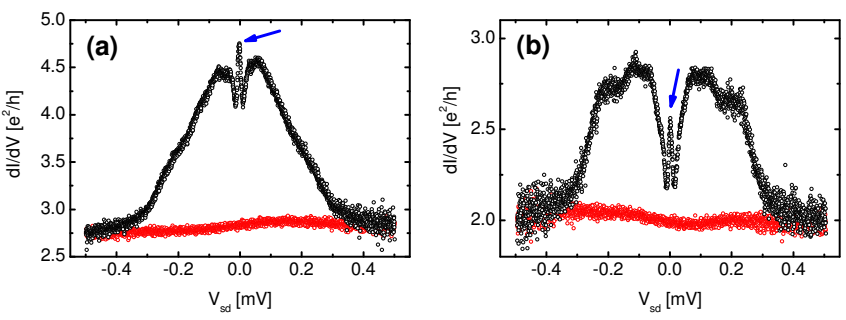

FIG. 3: (Color online) Differential conductance versus source drain voltage measured with lock-in amplifier $(5 \mu \mathrm{V}$ excitation) at different gate voltages as indicated in Fig. 2(c). Upper curve (black) is for the superconducting electrodes and lower curve (red) is with the magnetic field of $100 \mathrm{mT}$ to suppress the superconductivity of the electrodes. (a) is measured at a resonance of the Fabry-Perot pattern while (b) is measured at a antiresonance. Blue arrows point to the supercurrent peak.

current can flow providing that the interfaces are sufficiently transparent. In Fig. 3(b) we observe features for $\left|V_{\text {sd }}\right| \lesssim 2 \Delta / e$ and a distinct peak around zero bias, which is a general trend in our S-SWCNT-S junctions with high transparency. At $\left|V_{\mathrm{sd}}\right| \gg 2 \Delta / e$ transport is mostly due to quasiparticle transport and the FP pattern is seen in Fig. 2(b). As we approach $\left|V_{\mathrm{sd}}\right| \sim 2 \Delta / e$ from above the quasiparticle transport is enhanced due to the modified density of states of the superconductors and below this point a subharmonic gap structure (SGS) is expected to appear. We observe a complex pattern in the subgap region for the lower transparency case (Fig. 3(b)) while the higher transparency one (Fig. B(a)) doesn't show much structure in qualitative agreement with theoretical predictions 24, 25]. The structure in Fig. 3(b) seems too smeared to allow for quantitative comparison with theory, yet it is an interesting subject for further studies.

Instead of studying in detail the SGS, we focused on its integral effect in the form of the excess current, $I_{\text {exc }}$, which is defined as the difference in current between having the electrodes in the superconducting state and the normal state at $V_{\mathrm{sd}} \gg \Delta / e$. It can therefore be found as half of the difference in area between the two set of data points in Figs. 3(a-b). In Fig. 4(a), we have extracted the excess currents from the bias spectroscopy plot in Fig. 2(c) and plotted them as functions of the normal state differential conductance. Since the level width is much larger than the SC gap, $\Gamma \gg \Delta$, as can be seen from Figs. 2] 3 we can use for the interpretation of the results the well-established theory of superconducting (SC) point contacts [25, 26] and fit the excess current with the function

$$
I_{\mathrm{exc}}(g)=\frac{e \tilde{\Delta} g^{2}}{h(4-g)}\left[1-\frac{g^{2}}{4 \sqrt{4-g}(8-g)} \log \frac{2+\sqrt{4-g}}{2-\sqrt{4-g}}\right]
$$

where $g$ is the conductance measured in units of $e^{2} / h$ and
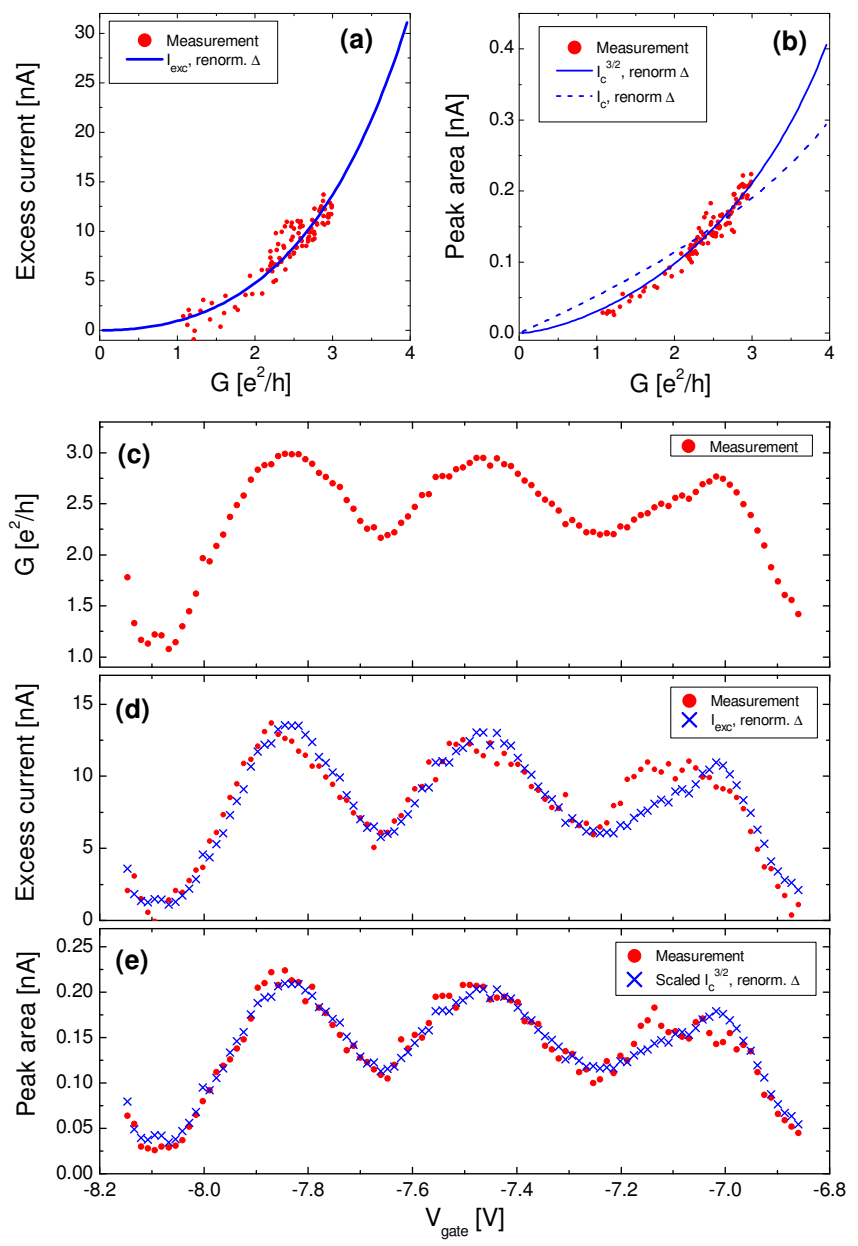

FIG. 4: (Color online) Comparison between measured and theoretical data. (a) Measured (dots) and fitted (line) excess current as a function of conductance $\left(\mathrm{G}=g e^{2} / h\right)$. The fitted line is calculated from Eq. (1) using $\tilde{\Delta}$ as a fitting parameter. (b) Measured (dots) and fitted (lines) supercurrent. The fits are to $a\left(I_{c}\right)^{3 / 2}$ (full line) and $a I_{c}$ (dashed line), using $a$ as a fitting parameter, see text for discussion. (c) Measured conductance in the Fabry-Perot regime as a function of gate voltage. (d) Measured (dots) and calculated (crosses) excess current. (e) The zero bias anomaly area measured (dots) and calculated (crosses). The theoretical points (crosses) in (d) and (e) are calculated using the measured conductance in (c) and Eqs. (1) and (2), respectively.

where $\tilde{\Delta}$ is the gap parameter at the superconductorCNT interface. Allowing for renormalization of $\tilde{\Delta}$ and performing a least-square fit to the measured data, we get $\tilde{\Delta} \sim 0.7 \Delta$. Using this value in Eq. (1) yields the curve in Fig. 廿(a) and also the data points in Fig. 廿(d), showing good agreement between experiments and the theoretically extracted excess current.

Next, we discuss the zero bias anomaly that we observe for all gate voltages in the FP-region. The peak has a full width of only $\sim 25 \mu \mathrm{V}$ (see Fig. 3). Such a peak in conductance at zero bias was observed earlier 
[14, 15] and was attributed to a dissipative quasiparticle current 27]. In this work, however, we pursue an alternative interpretation viewing the peak as a manifestation of the supercurrent. We interpret half of the area of the zero-bias peak (not the whole area under the peak) as a measure of the supercurrent. At first glace, this interpretation seem inconsistent since the measured peak area is of the order of $0.2 \mathrm{nA}$, while the expected magnitude of the supercurrent is on the order of $2 e \tilde{\Delta} / \hbar \sim 35 \mathrm{nA}$, i.e., more than two orders of magnitude more. However, a similar discrepancy between the measured and expected values of the supercurrent has been observed previously [9, 28]. In particular, in a very recent study [9] on a similar device to ours where the supercurrent was measured as zero bias current a discrepancy of the order of 15 between the measured and expected value was found. The suppression can be understood in terms of the dynamics of the SC phase of a resistively shunted Josephson junction with the environment, resulting in an apparent critical current $I_{m}$ scaling as $I_{m} \propto I_{c}^{3 / 2}$ with $I_{c}$ being the bare critical value of the supercurrent $I_{c}$, see Refs. 9, 28] for details [29].

Adopting this idea, we perform a fit to the measured zero-bias-peak area as a function of the normal state conductance, Fig. 4(b). The supercurrent is determined as $[24,26]$

$$
I_{c}(g)=\frac{e \tilde{\Delta} g \sin \varphi_{\max }}{4 \hbar \sqrt{1-\frac{g}{4} \sin ^{2}\left(\frac{\varphi_{\max }}{2}\right)}} \tanh \frac{\tilde{\Delta} \sqrt{1-\frac{g}{4} \sin ^{2}\left(\frac{\varphi_{\max }}{2}\right)}}{2 k_{B} T}
$$

where $\varphi_{\max }$ is the phase at which the supercurrent in Eq. (2) is maximal. Using the renormalized value of the gap, we have fitted the data to both $a I_{c}(g)$ and $a I_{c}^{3 / 2}(g)$ with $a$ being a fitting parameter. We clearly see that the conductance dependence of $I_{c}^{3 / 2}(g)$ fits the measured data very well. On the other hand the dependence of $I_{c}(g)$ doesn't fit the data at all, comparable with analogous results of Ref. [9]. The resulting $V_{\text {gate-dependence }}$ of the peak area is plotted in Fig. 廿4(e) using the fitted values of $I_{c}^{3 / 2}(g)$ with $\tilde{\Delta}$. Thus, we conclude that the zerobias-peak results are fully consistent with the theoretical predictions based on the supercurrent interpretation.

In conclusion, we have successfully fabricated gated SSWCNT-S Josephson junctions with high transparency contacts. In the Fabry-Perot regime of the semiconducting SWCNT reported here we observed quasiparticle tunneling at $\left|V_{\mathrm{sd}}\right|=2 \Delta / e$, enhanced current due to MARs for $\left|V_{\mathrm{sd}}\right|<2 \Delta / e$, and a conductance peak around zero bias. We interpret the zero bias conduction peak as a not fully developed supercurrent. The excess current, which has not been analyzed before for such junctions, fits very well to the theory of coherent SNS junctions.

We wish to acknowledge the support of the Danish Technical Research Council (The Nanomagnetism frame- work program), EU-STREP Ultra-1D program and the Nano-Science Center, University of Copenhagen, Denmark. The work of T. N. is a part of the research plan MSM 0021620834 that is financed by the Ministry of Education of the Czech Republic.

* Electronic address: hij@fys.ku.dk

[1] S. Tans, M. H. Devoret, H. Dai, A. Thess, R. E. Smalley, L. J. Geerligs, and C. Dekker, Nature 386, 474 (1997).

[2] M. Bockrath, D. H. Cobden, P. L. McEuen, N. G. Chopra, A. Zettl, A. Thess, and R. E. Smalley, Science 275, 1922 (1997).

[3] D. H. Cobden and J. Nygård, Phys. Rev. Lett. 89, 046803 (2002).

[4] P. J. Herrero, S. Sapmaz, C. D. L. P. Kouwenhoven, and H. S. van der Zant, Nature 429, 389 (2004).

[5] J. Nygård, D. H. Cobden, and P. E. Lindelof, Nature 408, 342 (2002).

[6] B. Babic, T. Kontos, and C. Schönenberger, Phys. Rev. B 70, 235419 (2004).

[7] W. Liang, M. Bockarth, D. Bozovic, J. H. Hafner, M. Tinkham, and H. Park, Nature 411, 665 (2001).

[8] J. Cao, Q. Wang, M. Rolandi, and H. Dai, Phys. Rev. Lett. 93, 216803 (2004).

[9] P. Jarillo-Herrero, J. A. van Dam, and L. P. Kouwenhoven, Nature 439, 953 (2006), cond-mat/0601434.

[10] J. Haruyama, K. Takazawa, S. Miyadai, A. Takeda, N. Hori, I. Takesue, Y. Kanda, N. Sugiyama, T. Akazaki, and H. Takayanagi, Phys. Rev. B 68, 165420 (2003).

[11] A. Y. Kasumov, R. Deblock, M. Kociak, B. Reulet, H. Bouchiat, I. I. Khodos, Y. B. Gorbatov, V. T. Volkov, C. Journet, and M. Burghard, Science 284, 1508 (1999).

[12] M. Tinkham, Introduction to superconductivity (McGraw-Hill Inc., ISBN, 0-07-064878-6, 1996), p. 201.

[13] A. F. Morpurgo, J. Kong, C. M. Marcus, and H. Dai, Science 286, 263 (1999).

[14] M. R. Buitelaar, W. Belzig, T. Nussbaumer, B. Babic, C. Bruder, and C. Schönenberger, Phys. Rev. Lett. 91, 057005 (2003).

[15] M. R. Buitelaar, T. Nussbaumer, and C. Schönenberger, Phys. Rev. Lett. 89, 256801 (2002).

[16] J. Kong, H. T. Soh, A. M. Cassell, C. F. Quate, and H.Dai, Nature 395, 878 (1998).

[17] K. Grove-Rasmussen, H. I. Jørgensen, and P. E. Lindelof, cond-mat/0601371 (2006).

[18] A.F.Andreev, Zh. Eksp Theor. Fiz. 46, 1823 (1964).

[19] G. E. Blonder, M. Tinkham, and T. M. Klapwijk, Phys. Rev. B 25, 4515 (1982).

[20] T. M. Klapwijk, M. Tinkham, and G. E. Blonder, Physica B+C 109-110, 1657 (1982).

[21] P. E. Gregers-Hansen, E. Hendricks, M. T. Levinsen, and G. R. Pickett, Phys. Rev. Lett. 31, 524 (1973).

[22] M. Octavio, M. Tinkham, G. E. Blonder, and T. M. Klapwijk, Phys. Rev. B 27, 6739 (1983).

[23] J. C. Cuevas and W. Belzig, Phys. Rev. B 70, 214512 (2004).

[24] A. Martín-Rodero, A. Levy Yeyati, and J. C. Cuevas, Superlattices and Microstructures 25, 925 (1999).

[25] J. C. Cuevas, A. Martín-Rodero, and A. L. Yeyati, Phys. 
Rev. B 54, 7366 (1996).

[26] G. Johansson, E. N. Bratus, V. S. Shumeiko, and G. Wendin, Phys. Rev. B 60, 1382 (1999).

[27] E. Vecino, M. R. Buitelaar, A. Martín-Rodero, C. Schönenberger, and A. L. Yeyati, Solid State Commun. 131, 625 (2004).
[28] P. Joyez, P. Lafarge, A. Filipe, D. Esteve, and M. H. Devoret, Phys. Rev. Lett. 72, 2458 (1994).

[29] The important parameter for the validity of this description is the quality factor $Q>1$ which is estimated to be 2 in our case [9]. 